\title{
Sources alimentaires et consommation estimée de CLA
}

\section{Nicole $\mathrm{COMBE}^{1}$ \\ Odile MORIN $^{2}$}

\author{
${ }^{1}$ ITERG Département de biochimie et nutrition, \\ c/o Université Bordeaux 1, Avenue des Facultés, \\ 33405 Talence Cedex \\ $<$ <.combe@istab.u-bordeaux1.fr> \\ 2 ITERG Coordination scientifique et technique, \\ rue Monge, 33600 Pessac
}

\begin{abstract}
The term "conjugated linoleic acid" (CLA) describes a group of geometrical and positional isomers of linoleic acid (18:2 9cis 12cis) with double bonds in conjugated position. These isomers are the 18:2 8trans 10cis, 18:29cis 11trans, 18:2 10trans 12cis and 18:211cis 13trans. In human diet, the fats from ruminants are the natural source of these fatty acids (milk, meat...). CLAs is produced by the rumen anaerobic bacteria metabolism of linoleic fatty acid, the 18:2 9cis 11trans being the predominant isomer (up to $90 \%$ of total CLAs), and named for that reason "rumenic acid".

The CLA richest food is milk (2 - $40 \mathrm{mg} / \mathrm{g}$ of fat, depending on the animal feed), as well as butter, dairy products, followed by meat of ruminants. Vegetable oils and margarine contain only small amounts of CLAs (0 - $0.5 \mathrm{mg} / \mathrm{g}$ ), originating from technological processes. Some significant quantities of CLAs are found in human breast milk, depending on women dietary habits (from 1.9 to $11.2 \mathrm{mg} / \mathrm{g}$ ).

The human consumption levels of CLAs have been estimated in different countries. With food questionnaires of the "3-7 days recall" or "semi-quantitative frequency" types, the population consumption has been estimated between 20 and 500 mg per day, with higher levels in men than in women. In Australia, the dietary intake may reach in some cases $1.5 \mathrm{~g} /$ day.
\end{abstract}

Key words: conjugated linoleic acid, dairy products, ruminants, vegetable oils, margarines, baby formula and food, daily intake plus riche. Il existe de nombreuses données sur le taux de CLA dans le lait de vache (tableau 1). Ce taux peut varier largement d'un lot à l'autre (de 2 à $37 \mathrm{mg} \mathrm{CLA} / \mathrm{g}$ de matière grasse) [3]. Parmi les facteurs qui influent sur la teneur en CLA du lait des ruminants, le plus important est le mode d'alimentation des animaux. Lorsque ceux-ci sont en pâturages, nourris d'herbe fraîche, la teneur en CLA du lait est 2 à 3 fois plus élevée que chez les animaux en stabulation, nourris d'aliments concentrés. Les acides gras polyinsaturés contenus dans l'herbe fraîche favorisent la biosynthèse ruminale de CLA. En conséquence, la teneur en CLA du beurre varie au cours de l'année. La figure 1 montre que le contenu en acide ruménique (18:29c 11t) des beurres d'hiver, lorsque les vaches sont en stabulation, diffère peu d'une région à l'autre, à savoir $0,34 \mathrm{~g} / 100 \mathrm{~g}$ de matière grasse en Aquitaine contre 0,4 $\mathrm{g}$ en Bretagne et en Normandie. En revanche, I'influence régionale est grande sur le contenu en acide ruménique des beurres d'été, période au cours de laquelle le taux de CLA augmente ; c'est en Normandie qu'il est notablement le plus élevé $(0,86 \mathrm{~g}$ contre $0,44 \mathrm{~g}$ en Aquitaine et $0,64 \mathrm{~g}$ en Bretagne) [6]. Le taux de CLA du lait peut également varier d'une vache à l'autre, entre 5 et 10 fois, pour un même mode d'alimentation. C'est alors la capacité plus ou moins grande de biosynthèse du $18: 29 \mathrm{c} 11 \mathrm{t}$, en relation avec le niveau d'activité de la $\Delta 9$ désaturase des tissus, qui pourrait expliquer ces différences.

Tous les produits laitiers, tels que le yaourt et les fromages, sont des aliments vecteurs de CLA. ruminants (boeuf, mouton, etc.) D'autres aliments peuvent en contenir en faible quantité (produits élaborés, viande d'animaux non ruminants, œufs, etc.). Au début des années 80, Pariza et al. [2] montrent que la viande de bœuf grillée contient à la fois des substances mutagènes et une substance, identifiée ensuite comme un CLA, qui inhibe la mutagenèse. Depuis ces premières observations, un grand nombre d'études a été consacré aux effets biologiques des CLA, dont certains sont développés dans les autres parties de ce dossier.

\section{Composition et teneur en CLA des aliments}

\section{Le lait et ses produits dérivés}

Le lait et les produits laitiers représentent, dans I'alimentation, la source commune de CLA la
Tableau 1. Teneurs en CLA de produits laitiers et viandes d'animaux ruminants.

\begin{tabular}{|c|c|c|}
\hline & $\%$ des acides gras totaux & références \\
\hline \multicolumn{3}{|l|}{ Lait et produits laitiers } \\
\hline \multirow[t]{2}{*}{ Laits (cru, pasteurisé, concentré) } & $0,5-1,1$ & Lin et al. [4] \\
\hline & & Fritsche et al. [5] \\
\hline Beurre & $0,5-0,8$ & Ledoux et al. [6] \\
\hline \multirow[t]{2}{*}{ Yaourt } & $0,4-0,7$ & Lin et al. [4] \\
\hline & & Fritsche et al. [5] \\
\hline \multirow[t]{2}{*}{ Comté } & $0,9-2,1$ & Fritsche et al. [5] \\
\hline & & Lavillonnière et al. [7] \\
\hline Emmental & $1,2-1,7$ & Fritsche et al. [5] \\
\hline \multicolumn{3}{|l|}{ Viandes de ruminants } \\
\hline \multirow[t]{2}{*}{ Bœuf } & $0,4-0,7$ & Fritsche et al. [8] \\
\hline & & Shantha et al. [9] \\
\hline \multirow[t]{2}{*}{ Agneau } & $0,6-1,2$ & Fritsche et al. [5] \\
\hline & & Chin et al. [10] \\
\hline
\end{tabular}




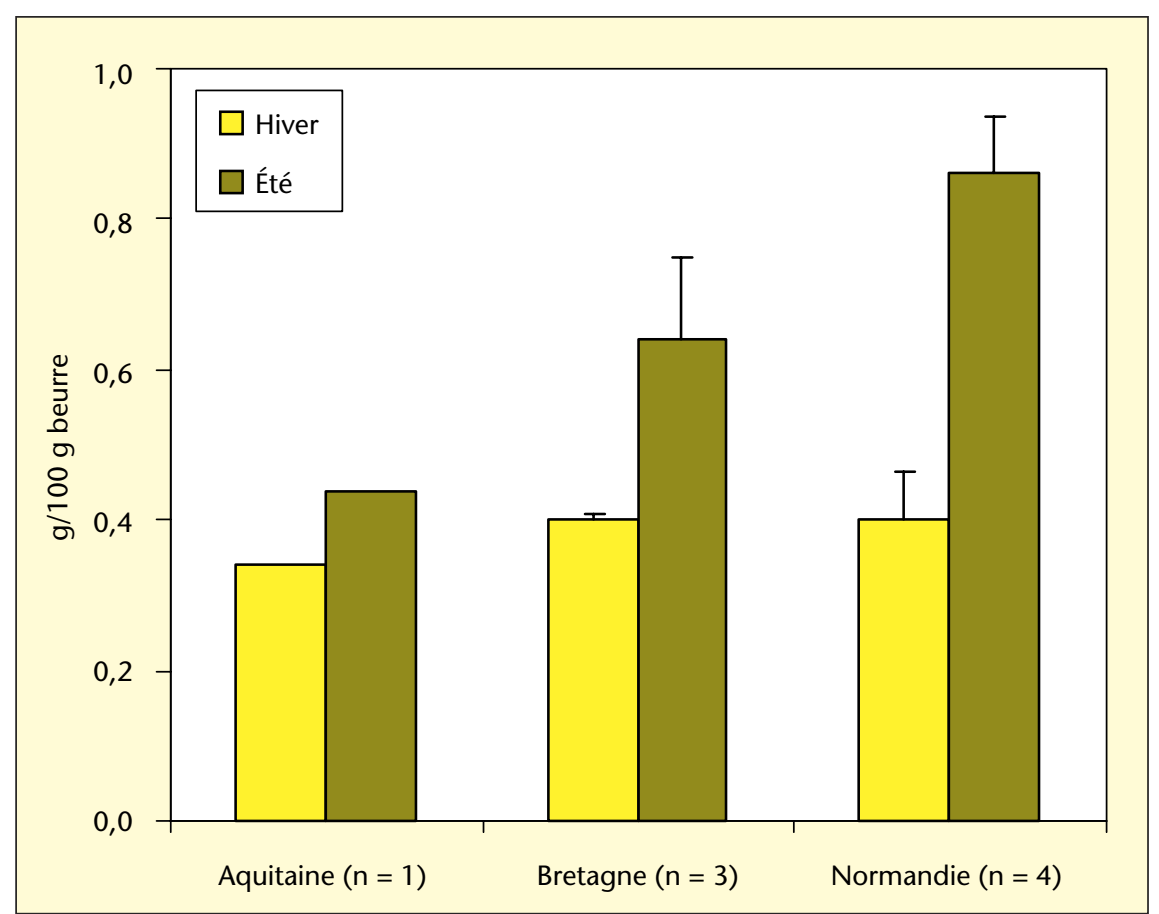

Figure 1. Variations saisonnières de la teneur en acide ruménique $(18: 29 \mathrm{c} 11 \mathrm{t})$ du beurre en fonction de l'origine géographique, d'après Ledoux $M$ et al. [6].

Comme dans le beurre, leurs teneurs en CLA varient notablement (tableau 1) en fonction de celles des laits d'origine. Le "Comté » est le fromage qui présente le taux de CLA le plus élevé (jusqu'à 2,1 \% de la matière grasse), effet attribué à la race des vaches (Montbéliard) qui produisent le lait destiné à ce fromage.

Le contenu en CLA des laits de chèvre et de brebis détermine également la teneur en CLA des fromages correspondants, dont la consommation est relativement importante, en particulier en France. Le lait de brebis a un taux de CLA similaire à celui du lait de vache $(1-3 \mathrm{~g}$ pour $100 \mathrm{~g}$ de matière grasse). En revanche, le lait de chèvre en contient 2 à 3 fois moins $(0,6-1,1 \mathrm{~g})$; on constate que la saison influe peu sur le taux de CLA du lait de chèvre, contrairement aux deux autres types de lait [11].

La composition en isomères CLA est disponible dans quelques études seulement [6-8], en raison des difficultés d'analyse inhérentes aux structures conjuguées de ces acides gras. Dans le beurre et les fromages, l'acide ruménique (9cis 11 trans) est très largement majoritaire (85 \%-93 \% des CLA totaux), il y a 6-9\% de CLA trans, trans et 1-5\% de CLA cis, cis. La figure 2 illustre les teneurs en CLA totaux et en acide ruménique de 18 beurres d'hiver, de printemps et d'été, issus de 7 régions françaises différentes. En moyenne, les proportions d'acide ruménique sont plus élevées dans les beurres d'été ( $93 \%$ des CLA totaux) que dans ceux d'hiver (89\%). Elles représentent également un vecteur important de CLA, entre $25 \%$ et $32 \%$ de I'apport alimentaire de CLA selon le pays [3]. Les teneurs en CLA des viandes de ruminants $(n=18)$, d'après Ledoux M et al. [6]. varient de $0,4 \mathrm{~g}$ à $1,2 \mathrm{~g} / 100 \mathrm{~g}$ de matière grasse (tableau 1). En moyenne, la viande de bœuf d'Australie contient plus de CLA, comparée à celles d'autres pays, dont la France. Le mode alimentaire des animaux influe sur le contenu en CLA de la viande comme sur celui du lait. Concernant la viande d'agneau, c'est la plus riche en CLA parmi les viandes de ruminants ; à titre d'exemple, le taux de CLA dans la viande d'agneau d'Australie est d'environ $1,5 \mathrm{~g} / 100 \mathrm{~g}$ de matière grasse versus $0,76 \mathrm{~g}$ pour la viande de bœuf de même origine géographique.

Concernant l'impact éventuel de la cuisson des viandes sur leurs teneurs en CLA, en particulier le mode de cuisson, aucune augmentation ni diminution n'ont été observées.

\section{Les viandes d'animaux non ruminants}

Les travaux de Fritsche et al. [5] montrent que les viandes de porc, de poulet ou encore de lapin contiennent de faibles teneurs en CLA $(0,1-0,2 \mathrm{~g} / 100 \mathrm{~g}$ de matière grasse), soit 2 à 12 fois moins que la viande de ruminants. La présence de CLA dans ces viandes pourrait trouver son origine dans l'alimentation des animaux (complémentation en suif, poudre de lait, etc.). Parmi les animaux non ruminants, seuls les marsupiaux possèdent des teneurs élevées en CLA (environ $3 \mathrm{~g} / 100 \mathrm{~g}$ de matière grasse), voire plus élevées que celles des animaux ruminants. Chez ces animaux, la présence de CLA est liée à leur processus digestif, proche de celui

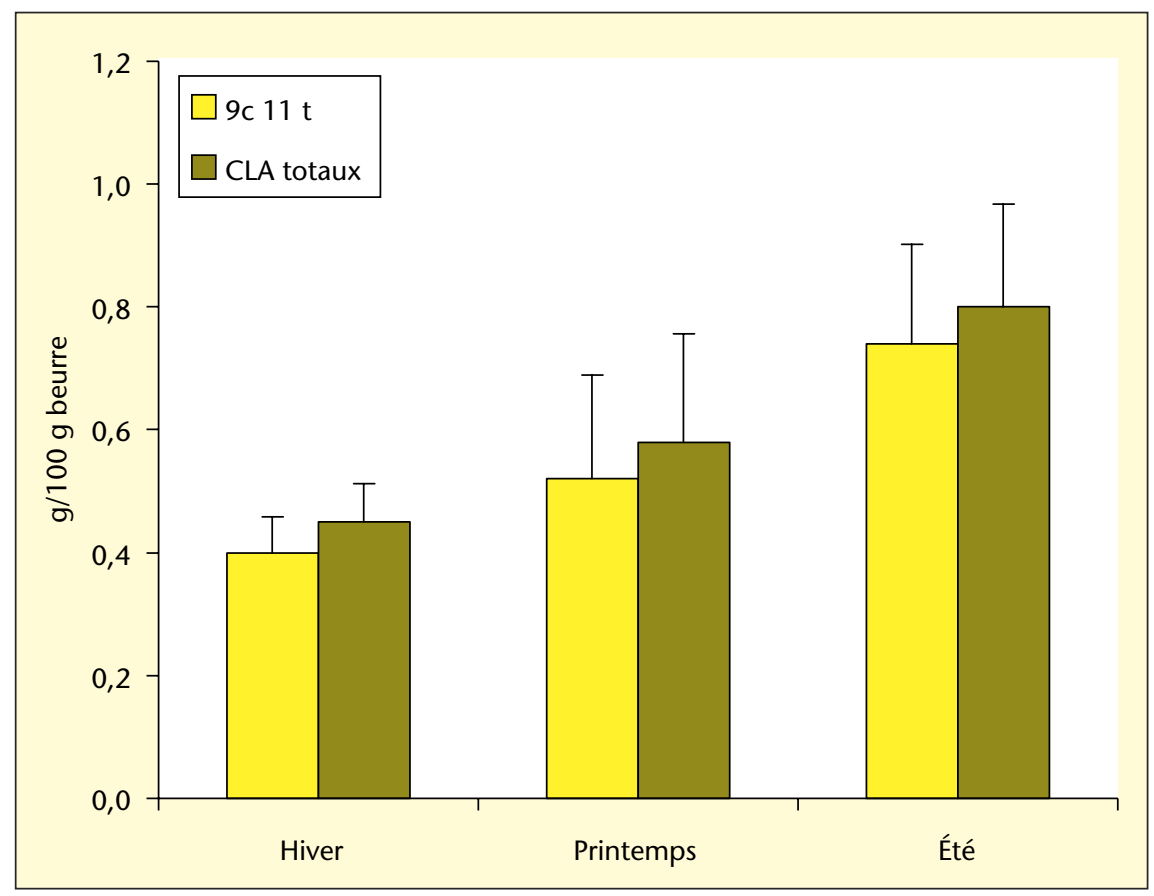

Figure 2. Variations saisonnières de la teneur en CLA totaux et en isomère 18 :2 9c 11t (acide ruménique) du beurre 
des ruminants. Dans les poissons, les CLA sont à l'état de traces.

\section{Les matières grasses végétales}

\section{Les huiles de table}

Le contenu en CLA des huiles est en général très faible ; les CLA peuvent se former lors des procédés technologiques, en particulier ceux qui mettent en jeu des températures élevées. Fritsche et al. [5] indiquent que les teneurs sont $<0,1 \mathrm{mg} / \mathrm{g}$, soit $100 \mathrm{ppm}$, dans 10 huiles de graines différentes. En revanche, Juanéda et al. [12] ont rapporté des taux compris entre 3 et $5 \mathrm{mg} / \mathrm{g}$ dans des huiles de tournesol, arachide et colza chauffées usagées. Dans ce cas, I'acide ruménique ne prévaut pas, d'autres isomères (en particulier $18: 2$ trans trans) sont en quantités notables.

\section{Les margarines}

Les taux de CLA dans les margarines de table sont très faibles, compris entre 0 et $0,5 \mathrm{mg} / \mathrm{g}$ de matière grasse [13]. Les margarines destinées aux produits manufacturés (shortenings) contiennent également des quantités négligeables $(0-0,9 \mathrm{mg} / \mathrm{g}$ de matière grasse).

\section{Les produits manufacturés}

\section{$\grave{A}$ base de viandes}

Les contenus en CLA de saucisses et de jambon reflètent ceux des viandes d'origine; ils sont compris entre 0,08 et $0,44 \mathrm{~g} / 100 \mathrm{~g}$ de matière grasse $[5,10]$.

\section{Gâteaux, pâtisseries, chocolat}

La teneur en CLA de ces produits est comprise entre $<0,01$ et $0,55 \mathrm{~g} / 100 \mathrm{~g}$ de matière grasse [5].

\section{Les aliments du nourrisson}

\section{Le lait maternel et les laits " maternisés "}

Une compilation des données issues de différents pays (Australie, Canada, États-Unis, Allemagne) est fournie dans la revue de Parodi [3]. Le contenu en CLA du lait de femmes peut varier notablement, de 1,8 à 11,2 mg par $\mathrm{g}$ de lipides. Cette large échelle des valeurs témoigne de l'impact des habitudes alimentaires des mères. La moyenne se situe autour de 4 à $6 \mathrm{mg}$ de CLA totaux par g de lipides. L'acide ruménique représente en moyenne $0,5 \%$ des acides gras totaux du lait maternel. Concernant les formules des laits "maternisés", le taux de CLA y est plus faible $(0,2 \mathrm{mg}-2,5 \mathrm{mg} / \mathrm{g}$ de lipides). Comme pour le lait maternel, l'isomère majeur dans ces formules est l'acide ruménique.
Tableau 2. Consommation estimée de CLA dans différents pays.

\begin{tabular}{|lcc|}
\hline Pays & Consommation (g/jour/pers) & Références \\
\hline Australie (1994) & $0,5-1,5$ & {$[14]$} \\
Allemagne (1998) & $0,35-0,43$ & {$[5]$} \\
Allemagne (2002) & $0,25-0,32$ & {$[15]$} \\
Suède (1999) & 0,16 & {$[16]$} \\
Pays-Bas (2002) & 0,2 & {$[17]$} \\
États-Unis (1998) & 0,13 & {$[18]$} \\
États-Unis (2001) & $0,10-0,18$ & {$[19]$} \\
- & $0,15-0,21$ & {$[19]$} \\
\hline
\end{tabular}

\section{Les préparations cuisinées}

La teneur en CLA des préparations cuisinées pour bébés varie très largement, en fonction de la nature des viandes entrant dans leur composition. Les préparations à base de volailles ont un contenu faible (de l'ordre de 0,02 $\mathrm{g}$ pour $100 \mathrm{~g}$ de matière grasse); dans les préparations à base d'agneau, le taux de CLA peut être multiplié par plus de 40 (soit $0,88 \mathrm{~g}$ pour $100 \mathrm{~g}$ de matière grasse).

\section{Consommation estimée de CLA}

La consommation de CLA a été estimée dans plusieurs pays (tableau 2), par diverses méthodes (questionnaires de fréquence alimentaire, relevé des consommations sur 24 h, 3 jours ou 7 jours) [5, 14-19]. Ces études montrent que les apports en CLA sont plus élevés chez les hommes que chez les femmes. Le niveau global d'apport en CLA le plus faible est observé aux États-Unis (0,1-0,2 g CLA/jour/personne) [18, 19]. En Europe, il apparaît plus élevé (0,2$0,4 \mathrm{~g})[5,15-17]$. II est néanmoins inférieur à celui rapporté pour l'Australie $(0,5-1,5 \mathrm{~g})$, où les produits $d^{\prime}$ animaux ruminants sont plus riches en CLA que dans les autres pays [14].
La figure 3 illustre une étude conduite aux États-Unis chez 48 hommes et 47 femmes, portant sur les contributions des aliments à l'apport journalier en CLA totaux et en acide ruménique [18]. Les résultats confirment la nature des principaux aliments vecteurs de CLA, qui assurent $92 \%$ de l'apport en CLA totaux. II s'agit des produits de ruminants, à savoir produits laitiers $(60 \%)$ et viande de bœuf (32\%). S'agissant de l'apport en acide ruménique, la situation comme attendue est similaire, à savoir $93 \%$ de l'acide ruménique consommé sont fournis par ces mêmes aliments, produits laitiers $(68 \%)$ et viande de bœuf (25\%).

\section{RÉFÉRENCES}

1. HA YL, GRIMM NK, PARIZA MW. Anticarcinovatives of linoleic acid. Carcinogenesis $1987 ; 8$ : 1881-7.

2. PARIZA MW, HARGRAVES WA. A beef-derived mutagenesis modulator inhibits initiation of mouse epidermal tumors by 7 , 12-dimethylbenzo [a] anthracene. Carcinogenesis $1985 ; 6: 591-3$. gens from fried ground beef : heat altered deri-

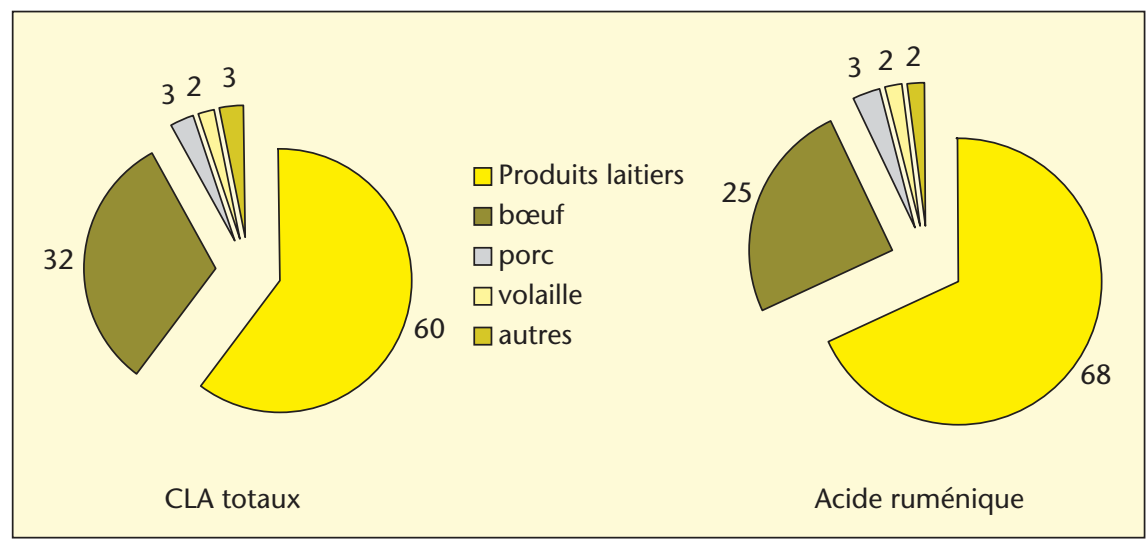

Figure 3. Contributions (\% de l'apport total) de divers aliments à l'apport journalier en CLA totaux et en acide ruménique d'une population (48 hommes et 47 femmes), d'après Ritzenthaler et al [18]. 
3. PARODI PW. Conjugated Linoleic acid in food In : Sébédio JL, Christie WW, Adlof RO, eds. Advances in Conjugated Linoleic Acid research. Vol. 2. Champaign, IL: AOCS Press, 2003 : 101-22.

4. LIN H, BOYLSTON T, LUEDECKE L, SHULTZ T. Survey of the Conjugated Linoleic Acid Contents of Dairy Products. I Dairy Sci 1995; $78: 2358-65$.

5. FRITSCHE J, STEINHART H. Amounts of conjugated linoleic acid (CLA) in german foods and evaluation of daily intake. $Z$ Lebensm Unters Forsch A $1998 ; 206$ : 77-82.

6. LEDOUX M, CHARDIGNY JM, DARBOIS M et al. Variations saisonnières des taux d'acides linoléiques conjugués dans les beurres français. Sci Alim $2003 ; 23: 443-61$.

7. LAVILLONNIÈRE F, MARTIN JC, BOUGNOUX P, et al. Analysis of conjugated linoleic acid isomers and content in French cheeses. / Am Oil Chem Soc $1998 ; 75$ : 343-52.

8. FRITSCHE I, FRITSCHE S, SOLOMON MB, et al. Quantitative determination of conjugated linoleic acid isomers in beef fat. Eur / Lipid SCi Technol $2000 ; 102: 667-72$.
9. SHANTHA NC, DECKER EA, USTUNOL Z Conjugated Linoleic Acid Concentration in Processed Cheese. I Am Oil Chem Soc 1992; 69 : 425-8.

10. CHIN SF, LIUW, STORKSON JM, HAYL, PARIZA MW. Dietary sources of conjugated dienoic isomers of linoleic acid, a newly recognized class of anticarcinogens. / Food Compos Anal $1992 ; 5$ : 185-97.

11. JAHREIS G, FRITSCHE J, MOCKEL $P$, et al. The potential anticarcinogenic CLA, cis 9, trans 11 $\mathrm{C} 18: 2$, in milk of different species : cow, goat, ewe, sow, mare, woman. Nutr Res $1999 ; 19$ : 1541-9.

12. JUANÉDA P, CORDIER O, GRÉGOIRE S, et al. Conjugated linoleic acid (CLA) isomers in heattreated vegetable oils. OCL $2001 ; 8$ : 94-7.

13. PRECHT D, MOLKENTIN J. Recent trends in the fatty acid composition of German sunflower margarines, shortenings and cooking fats with emphasis on individual C16:1, C18:1, C18:2, $\mathrm{C} 18: 3$ and $\mathrm{C} 20: 1$ trans isomers. Nahrung $2000 ; 4: 222-8$.

14. PARODI PW. Conjugated linoleic acid : an anticarcinogenic fatty acid present in milk fat. Aust J Dairy Technol 1994 ; 49 : 93-7.
15. FREMANN D, LINSEISEN J, WOLFRAM G. Dietary Conjugated linoleic acid (CLA) intake assessment and possible biomarkers of CLA intake in young women. Public Health Nutr $2002 ; 5: 73-80$.

16. JIANG J, WOLK A, VESSBY B. Relation between the intake of milk fat and the occurence of conjugated linoleic acid in human adipose tissue. Am / Clin Nutr 1999 ; 70 : 21-7.

17. WOORRIPS LE, BRANTS H, KARDINAAL AFM, et al. Intake of Conjugated linoleic acid, fat, and other fatty acids in relation to postmenopausal breast cancer : the Netherlands Cohort Study on Diet and Cancer. Am / Clin Nutr 2002 ; $76: 873-82$.

18. HERBEL BK, MCGUIRE MK, MCGUIRE MA, et al. Safflower oil consumption does not increase plasma conjugated linoleic acid concentrations in Humans. Am / Clin Nutr 1998 ; 67 : 332-7.

19. RITZENTHALER KL, MCGUIRE MK, FALEN R Estimation of conjugated linoleic acid intake by written dietary assessment methodologies underestimates actual intake evaluated by food duplicate methodology. I Nutr 2001; 131 : 1548-54. 